\title{
Supplementary Information: Oligomerization of Dehydrogenated Polycyclic Aromatic Hydrocarbons on Highly Oriented Pyrolytic Graphite (HOPG)
}

\author{
Jürgen Weippert ${ }^{1,2}$, Jakob Hauns ${ }^{1}$, Julian Bachmann ${ }^{1}$, Jean-Francois Greisch ${ }^{3}$, \\ Akimitsu Narita $^{4}$, Klaus Müllen ${ }^{4}$, Artur Böttcher ${ }^{1}$, and Manfred M. Kappes ${ }^{1,5}$ \\ ${ }^{1}$ Institute of Physical Chemistry, KIT, 76131 Karlsruhe, Germany \\ ${ }^{2}$ Fraunhofer IAF, Fraunhofer Institute for Applied Solid State Physics, 79108 Freiburg \\ im Breisgau, Germany \\ ${ }^{3}$ Biomolecular Mass Spectrometry and Proteomics, Utrecht University, 3584CH \\ Utrecht, Netherlands \\ ${ }^{4}$ Max Planck Institute for Polymer Research, 55128 Mainz, Germany \\ ${ }^{5}$ Institute of Nanotechnology, KIT, 76344 Eggenstein-Leopoldshafen, Germany
}

\section{Preparative ion beam compo- sition tuning}

The ion fluxes necessary to deposit sufficient amounts for TPD measurements could only be realized with comparatively wide mass filter transmission windows, i.e. at low mass resolution. This was sufficient to resolve $\mathrm{CH}$ loss, if desired, but not $\mathrm{nH}$ loss from the $\mathrm{PAH}^{+}$parent ions. Consequently, we deposited mixtures of parent and dehydrogenated fragment ions, $\mathrm{PAH}-\mathrm{nH}^{+}$, in all experiments reported here. It was nevertheless possible to tune the composition of deposited ions somewhat by varying the instrumental mass filter (= center mass) and mass resolution settings. In general we chose a mass filter setting corresponding to a center mass $2-8 \mathrm{u}$ below that of the parent ion. Furthermore, mass resolution was typically set to allow transmission of ions $\pm 5 \mathrm{u}$ around this center mass.

We illustrate this in figure S1 for the example of perdeuterated coronene ionized at an electron impact energy of $70 \mathrm{eV}$. The relative abundance of the ionic species contributing to the mass spectrum can be determined to a good approximation by fitting the mass spectrum with four different contributing

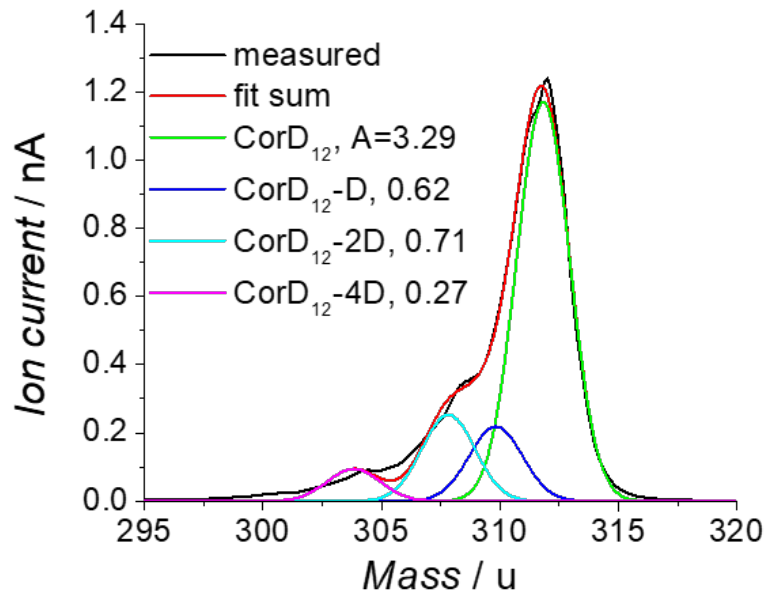

Figure S1: Mass spectrum of $\mathrm{CorD}_{12}$ in the preparative ion beam with $70 \mathrm{eV}$ impact energy fitted with Gaussian components in order to determine the fraction of dehydrogenated fragments

ionic species each of halfwidth $2.5 \mathrm{u}$. Small deviations between the sum of fitted Gaussians and the actual mass spectrum result from minor additional isotopic contributions as well as from the fact 


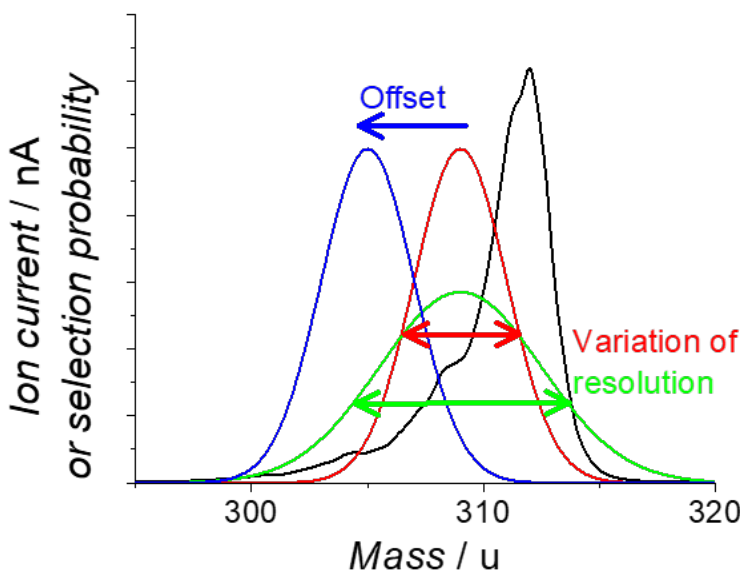

Figure S2: Visualization of the tuning process: the mass spectrometer selects a certain mass range of the beam (Gaussian function only for visualization, real function unknown); the degree of dehydrogenation in the deposited beam can be tuned by varying the offset (blue) or the resolution (red/green).

that the instrumental peak form is not fully Gaussian. According to this fit the ratio of dedeuterated species (integrated between 297.5 and $317.5 \mathrm{u}$ ) and parent ion is 1:2.1 in good agreement with the better resolved Massbank spectrum ${ }^{1}$ (at the same electron impact energy) for which a ratio $1: 2.5$ can be determined.

Figure S2 schematically illustrates the effect of changing instrumental mass offset (blue) and the resolution (red/green, $\Delta M=\Delta$ Res setting in the mass spectrometer software). The exact shape of the transmission function and how it depends on ion flux, ion mass and the instrumental resolution setting is unknown. Therefore the Gaussian shapes superimposed on the mass spectra are only meant to serve as visualization. Nevertheless, we believe them to qualitatively reflect the actual situation pertaining here.

\section{Theoretical densities of states of Coronene dimers}

In order to explore whether it might be possible to characterize the linking motif in dimers of coronene

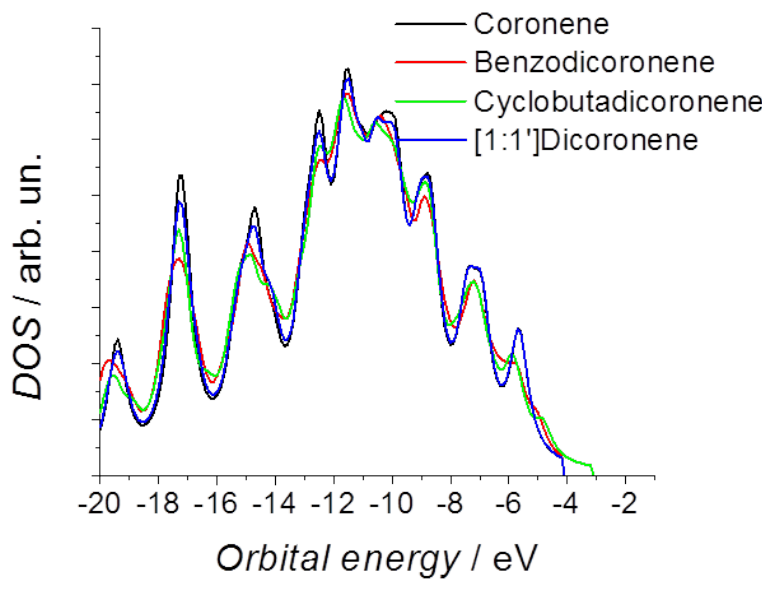

Figure S3: Theoretical densities of states for coronene and various coronene dimers (see main text) as generated by folding DFT orbital energies with Lorentzian functions of $0.75 \mathrm{eV}$ halfwidth. Monomers and dimers are basically indistinguishable at our experimental UPS resolution.

or other PAHs by acquiring Ultraviolet Photoemission Spectra (UPS), we simulated such spectra using DFT-calculated densities of states (DOS) determined by folding orbital energies of the most stable dimers with Lorentzian functions of halfwidth $0.75 \mathrm{eV}$. As we have recently shown this approach as applied to monomers leads to decent agreement with UP spectra (see supplements of our previous work $^{2,3}$ ). However, as fig. S3 clearly shows, the theoretical DOS of Coronene monomer and dimers are much too similar to allow for an assignment at the $<750 \mathrm{meV}$ UPS instrumental energy resolution achievable in our setup.

\section{Calculated structures of de- hydrogenated fragments and dimers}

The following figures S4-S8 show all structures we calculated for dehydrogenated fragments as well as for the resulting dimers. As described in the main paper, the level of DFT was always RI-B3LYP ${ }^{4-7}$ with the def2-TZVP ${ }^{8,9}$ basis set which, in the case of Rubrene, was supplemented by the Grimme-D3 correction ${ }^{10}$ in order to account for interactions between the Phenyl rings. 

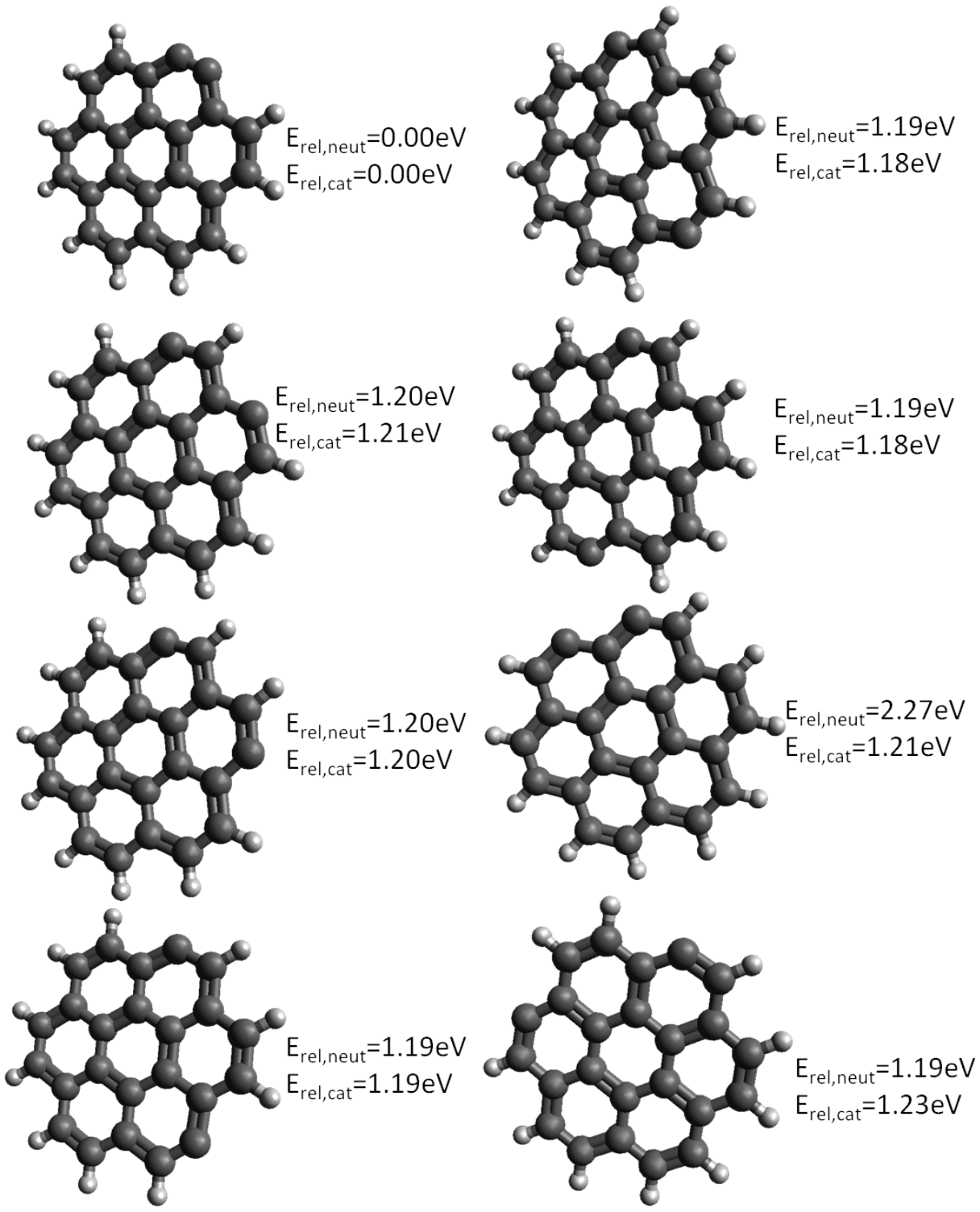

Figure S4: Calculated structures of Cor-2H with their relative energies both for cations and neutral molecules; in both charge states the aryne-like fragment is clearly the most stable one

All energies provided in these figures are relative energies compared to the most stable isomer of that stoichiometry in neutral (rel,neut) or cationic (rel,cat) charge state. Our calculations of dehydrogenated coronene fragments (fig. S4) gave the same result as in previous studies ${ }^{11}$ : the most stable fragment is the aryne-like fragment for which both hydrogen atoms are removed from the same Benzene ring.
The indices $n-m$ under the coronene dimers in figs. $\mathrm{S} 5+\mathrm{S} 6$ are meant to be read as follows: $\mathrm{n}$ is the number of sigma bonds in the interlinking unit while $m$ is the number of nominal double bonds within the same area. The dimers of pentacene ((fig. S7) do not show strong structural trends apart from the rather intuitive finding that the strongly bent dimer with two interlinking pentagons is extremely unstable in comparison with the almost 

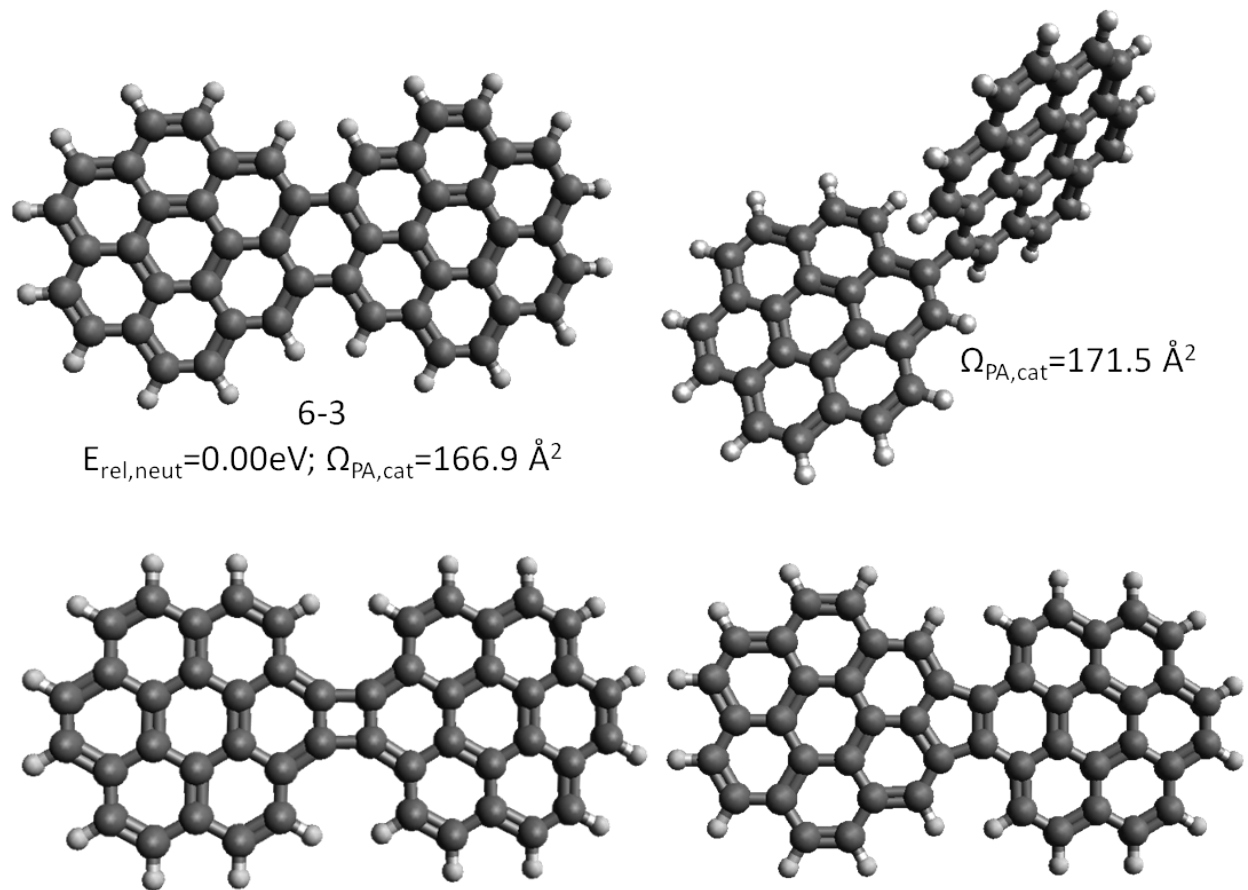

4-2

$E_{\text {rel, neut }}=2.54 \mathrm{eV} ; \Omega_{\mathrm{PA}, \text { cat }}=168.5 \AA^{2}$

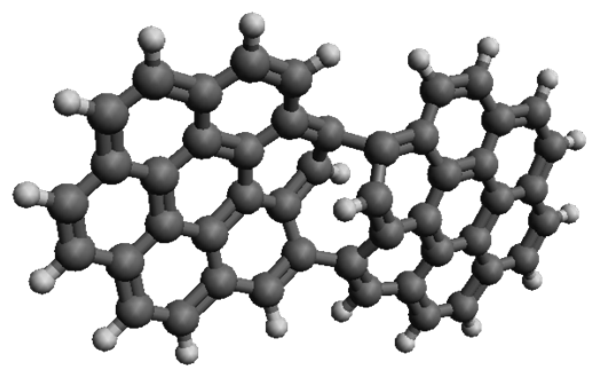

8-2a

$E_{\text {rel, neut }}=6.18 \mathrm{eV} ; \Omega_{\mathrm{PA}, \text { cat }}=163.5 \AA^{2}$

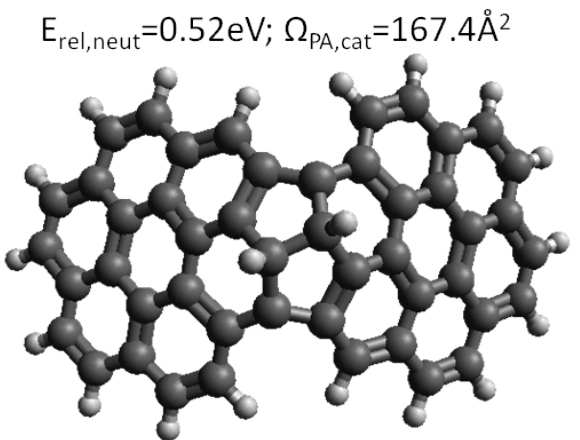

$8-2 b$

$E_{\text {rel, neut }}=4.91 \mathrm{eV} ; \Omega_{\mathrm{PA}, \text { cat }}=164.5 \AA^{2}$

Figure S5: Calculated structures of dimers $[\mathrm{Cor}-2 \mathrm{H}]_{2}$ annelled by small rings with relative stabilities of neutral molecules as well as theoretical cross sections of cations in projection approximation

planar structures.

Our calculations for DCY (fig. S8) did not reveal strongly preferred dimer structures, i.e. many dimer structures were found to be roughly equally stable. Regarding rubrene our calculations (also fig. fig. S8) showed that the stability differs significantly between cations and neutral molecules: while for cations the dehydrogenation of one of the Phenyl rings is strongly favored, in the neutral charge state the most stable fragment is dehydrogenated at the tetracene unit. 


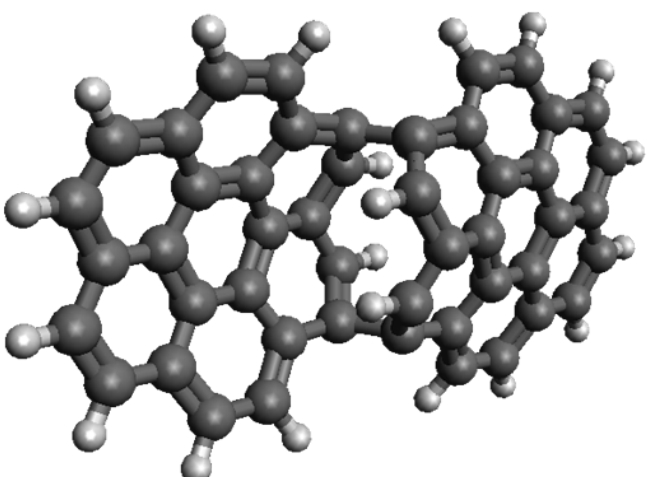

$10-4 a$

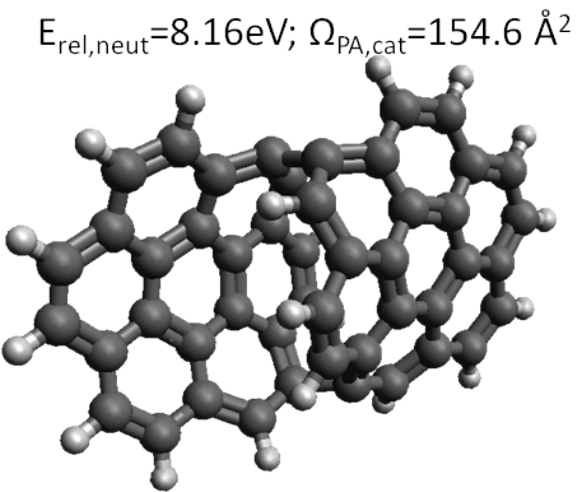

14-6a

$E_{\text {rel, neut }}=7.18 \mathrm{eV} ; \Omega_{P A, \text { cat }}=144.5 \AA^{2}$

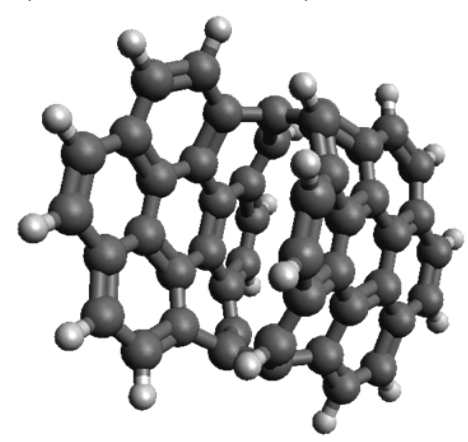

$16-8 a$

$E_{\text {rel, neut }}=8.28 \mathrm{eV} ; \Omega_{\mathrm{PA}, \text { cat }}=140.0 \AA^{2}$

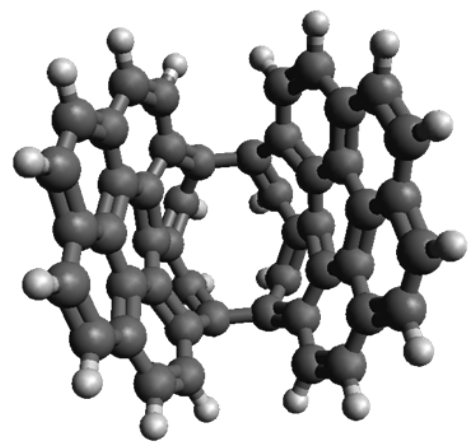

$10-4 a$

$E_{\text {rel, neut }}=9.24 \mathrm{eV} ; \Omega_{\mathrm{PA}, \text { cat }}=146.4 \AA^{2}$

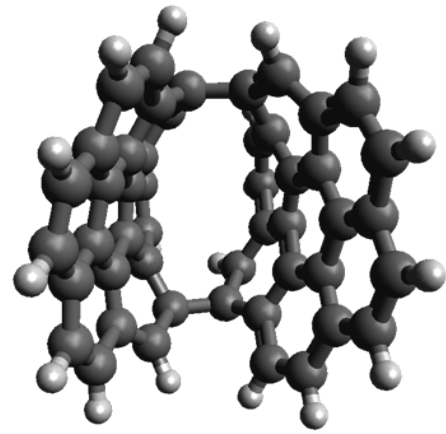

$14-6 b$

$E_{\text {rel, neut }}=7.96 \mathrm{eV} ; \Omega_{\mathrm{PA}, \text { cat }}=144.0 \AA^{2}$

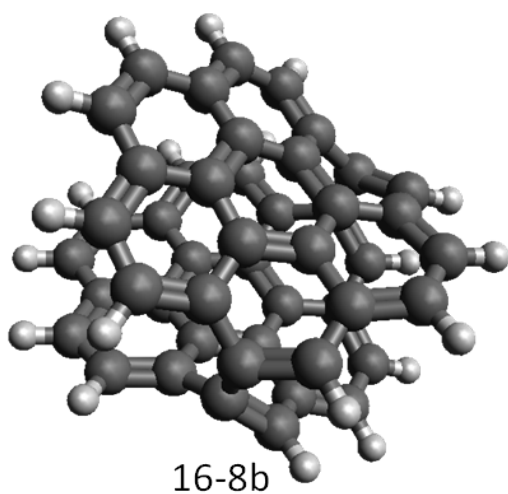

$E_{\text {rel, neut }}=8.35 \mathrm{eV} ; \Omega_{\mathrm{PA}, \text { cat }}=136.7 \AA^{2}$

Figure S6: Calculated structures of dimers $[\mathrm{Cor}-2 \mathrm{H}]_{2}$ annelled by large rings with relative stabilities of neutral molecules as well as theoretical cross sections of cations in projection approximation 

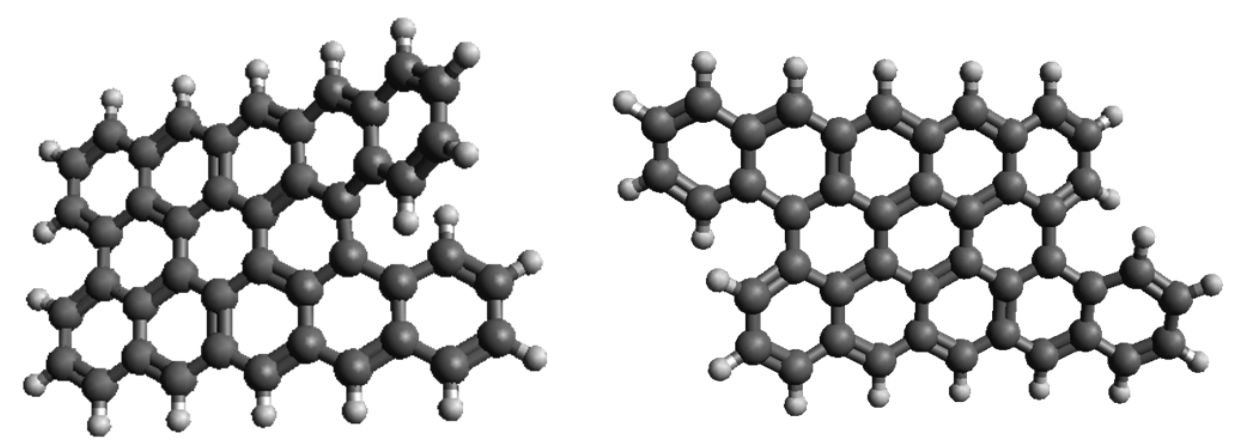

$$
E_{\text {rel }}=0.00 e V
$$
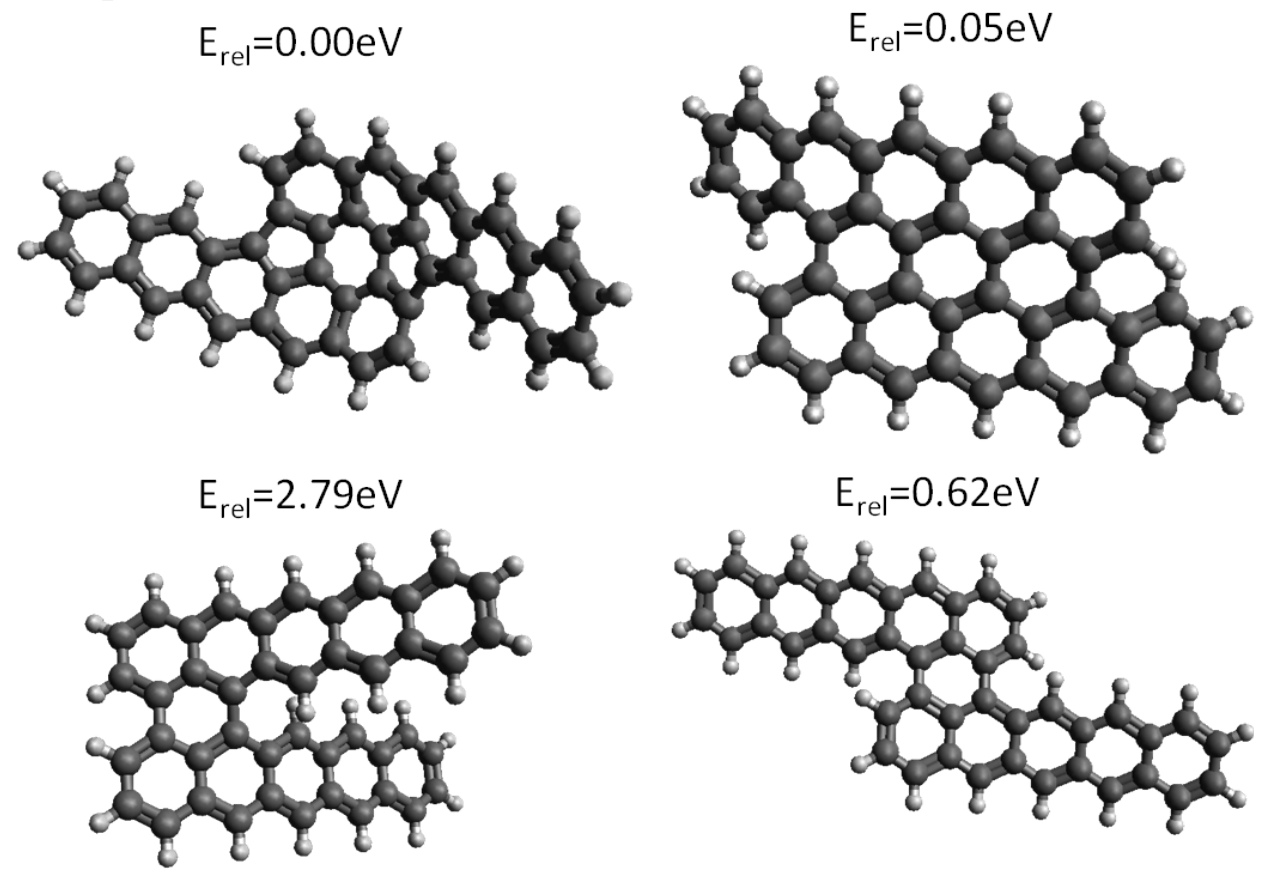

$$
E_{\text {rel }}=0.00 e V
$$$$
E_{\text {rel }}=0.15 \mathrm{eV}
$$

Figure S7: Calculated structures of dimers $[\mathrm{Pen}-4 \mathrm{H}]_{2}$ and $[\mathrm{Pen}-2 \mathrm{H}]_{2}$ with the relative stabilities of neutral molecules 


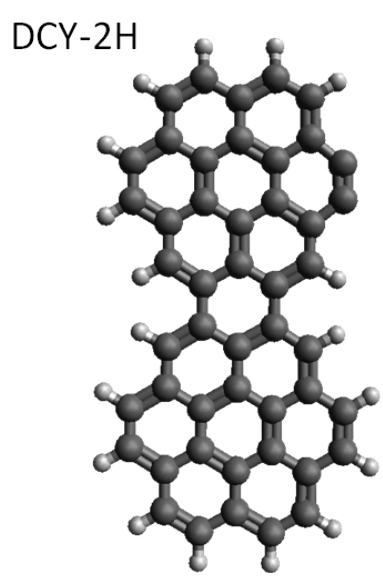

$E_{\text {rel,cat }}=0.00 \mathrm{eV}$

$\mathrm{E}_{\text {rel, neut }}=0.00 \mathrm{eV}$

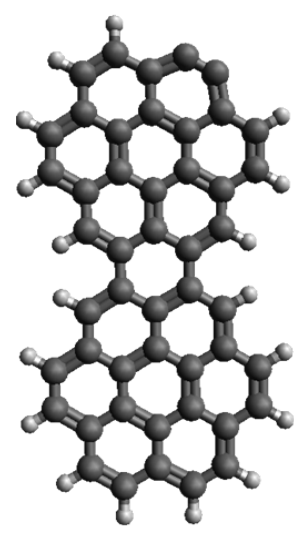

$\mathrm{E}_{\text {rel,cat }}=0.03 \mathrm{eV}$

$E_{\text {rel, neut }}=0.01 \mathrm{eV}$

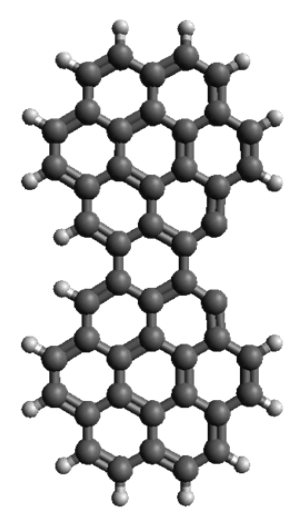

$\mathrm{E}_{\mathrm{rel}, \mathrm{cat}}=1.05 \mathrm{eV}$

$E_{\text {rel, neut }}=1.64 \mathrm{eV}$

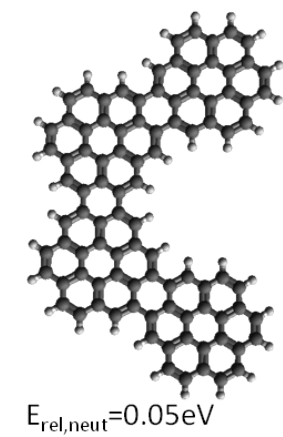

[DCY-2H $]_{2}$

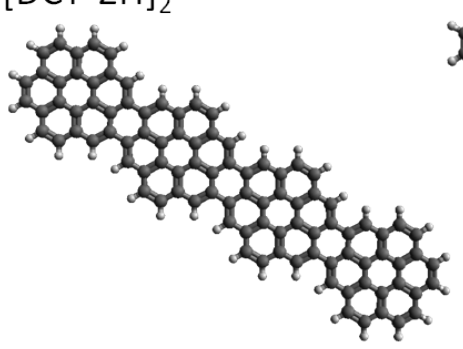

$E_{\text {rel,neut }}=0.00 \mathrm{eV}$
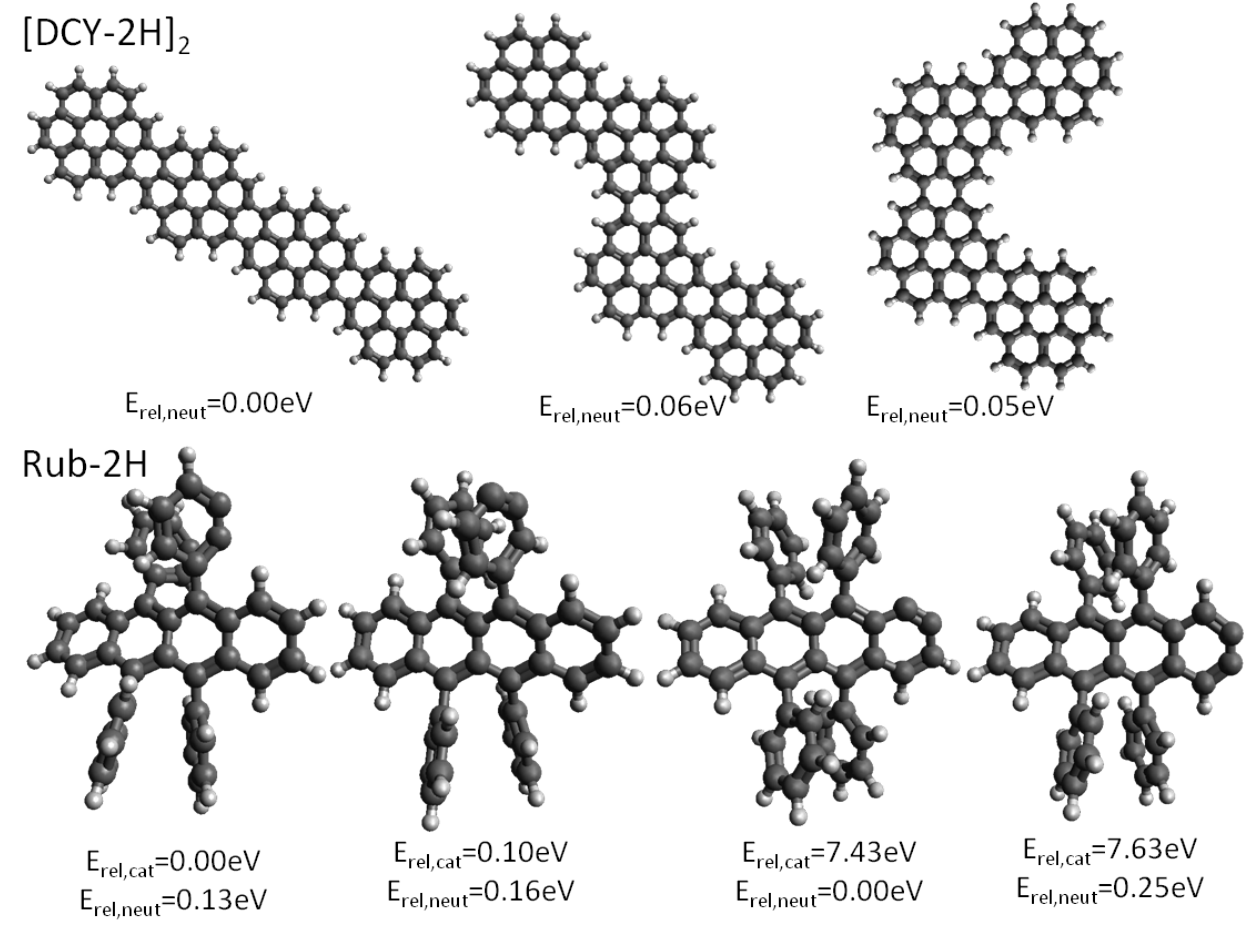

Figure S8: Calculated structures of dehydrogenated fragments DCY-2H and Rub-2H with their relative stabilities both as cations and as neutral molecules as well as three DCY dimers with their relative stabilities (neutral only) 


\section{Deposition experiments with rubrene fragments}

Rubrene with its branched structure has an additional potential fragmentation channel compared to planar PAHs: the loss of one of its Phenyl rings. Fig. S9 shows clearly that this is a strongly favored process that does not require too much electron impact energy. In addition to the loss of the Phenyl rings, the concerted loss of hydrogen atoms and of $\mathrm{CH}$ groups is also possible. We deposited both fragment groups Rub- $\mathrm{Ph}^{+}$and Rub- $2 \mathrm{Ph}^{+}$onto HOPG and analyzed the desorption of monomers. The desorption of dimers was never observed which suggests that both fragment groups are dominated by non-radical species.

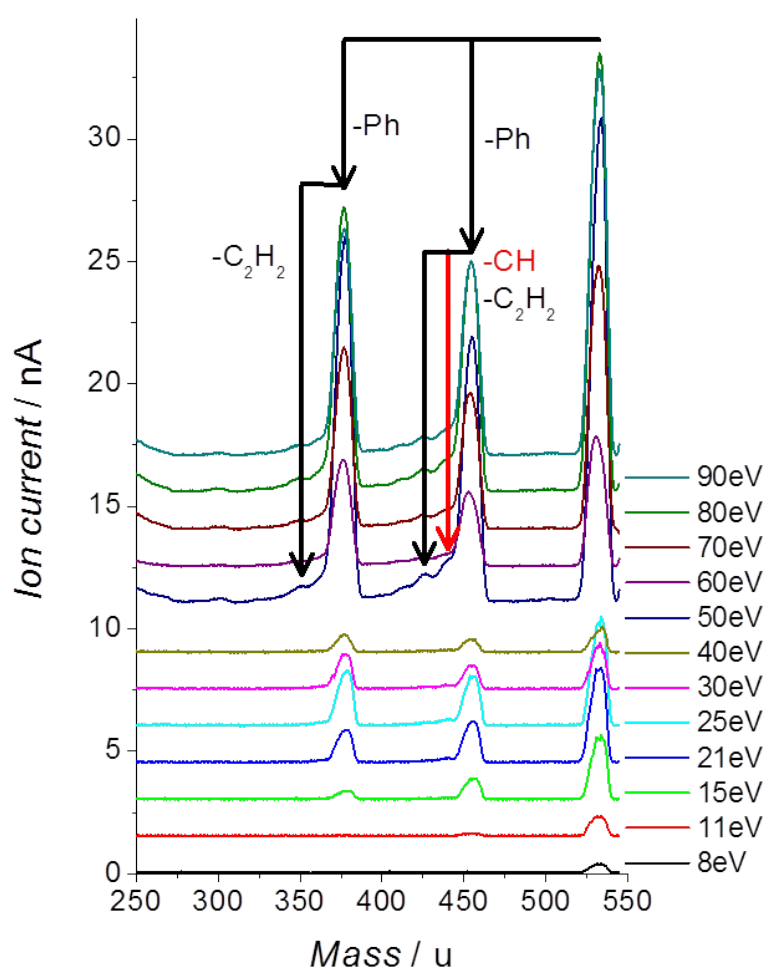

Figure S9: Mass spectra of rubrene as function of the electron impact energy with loss channels (note that individual hydrogen losses within the peaks are not resolved).

Rub- $\mathrm{Ph}-\mathrm{nH}$ desorbs in the temperature range 400$700 \mathrm{~K}$. As fig. $\mathrm{S} 10 \mathrm{~A}+\mathrm{B}$ shows, the signal is already divided into three components for rather low ion doses. At an ion dose of $1.84 \cdot 10^{13}$ ions $/ \mathrm{cm}^{2}$ an additional multilayer peak appears - at a much lower coverage than seen for rubrene ${ }^{3}$ indicating that the fragment films are not as densely packed as a rubrene film. This may in part be due to the fact that the fragments can be closer to a planar geometry and therefore require more space on the surface.

A closer analysis of TRMS and TPD coverage variation series shows that there are two Rub- $\mathrm{Ph}-\mathrm{H}$ components and one Rub-Ph-3H component. Our DFT calculations (see next section) provide a simple explanation for this: In the cationic states there are two equally stable Rub-Ph-H isomers while for Rub-Ph-3H there is one preferred isomer. The deposition of the fragment group Rub-2Ph-nH provides similar results (fig. $\mathrm{S} 9 \mathrm{C}+\mathrm{D}$ ): two components are already observed for very low ion doses while a multilayer signal appears basically at the same dose as in the case the Rub-Ph-nH group. In this case the monolayer signal consists of one $\mathrm{Rub}-2 \mathrm{Ph}$ and one Rub-2Ph-2H component of which the latter clearly dominates. This is also well- explainable using our DFT calculations: for both compositions there is one strongly preferred structure but the Rub- $2 \mathrm{Ph}$ geometry requires an intramolecular hydrogen transfer in the gas phase so this fragmentation channel is strongly disfavored. We can use the peak temperatures $\mathrm{T}_{P}$ to determine the binding energies $\mathrm{E}_{A}$ ( $\approx$ desorption activation energies) of all monolayer components. A Redhead analysis using the frequency factor $10^{17} \mathrm{hz}$ that we also used for rubrene binding energies ${ }^{3}$ gives the gives the numbers displayed in table $\mathrm{S} 1$.

\begin{tabular}{|l|l|l|}
\hline [Rub-mPh-nH] & $\mathrm{T}_{p} / \mathrm{K}$ & $\mathrm{E}_{A} / \mathrm{eV}$ \\
\hline $\mathrm{m}=1, \mathrm{n}=1(1)$ & 544 & 1.89 \\
$\mathrm{~m}=1, \mathrm{n}=1(2)$ & 584 & 2.04 \\
$\mathrm{~m}=1, \mathrm{n}=3$ & 646 & 2.26 \\
$\mathrm{~m}=2, \mathrm{n}=0$ & 516 & 1.79 \\
$\mathrm{~m}=2, \mathrm{n}=2$ & 572 & 1.99 \\
\hline
\end{tabular}

Table S1: Peak positions and values for $\mathrm{E}_{A}$ as determined by a Redhead analysis for all monolayer compounds of type [Rub-mPh-nH], assuming a frequency factor of $10^{17} \mathrm{~Hz}$. 

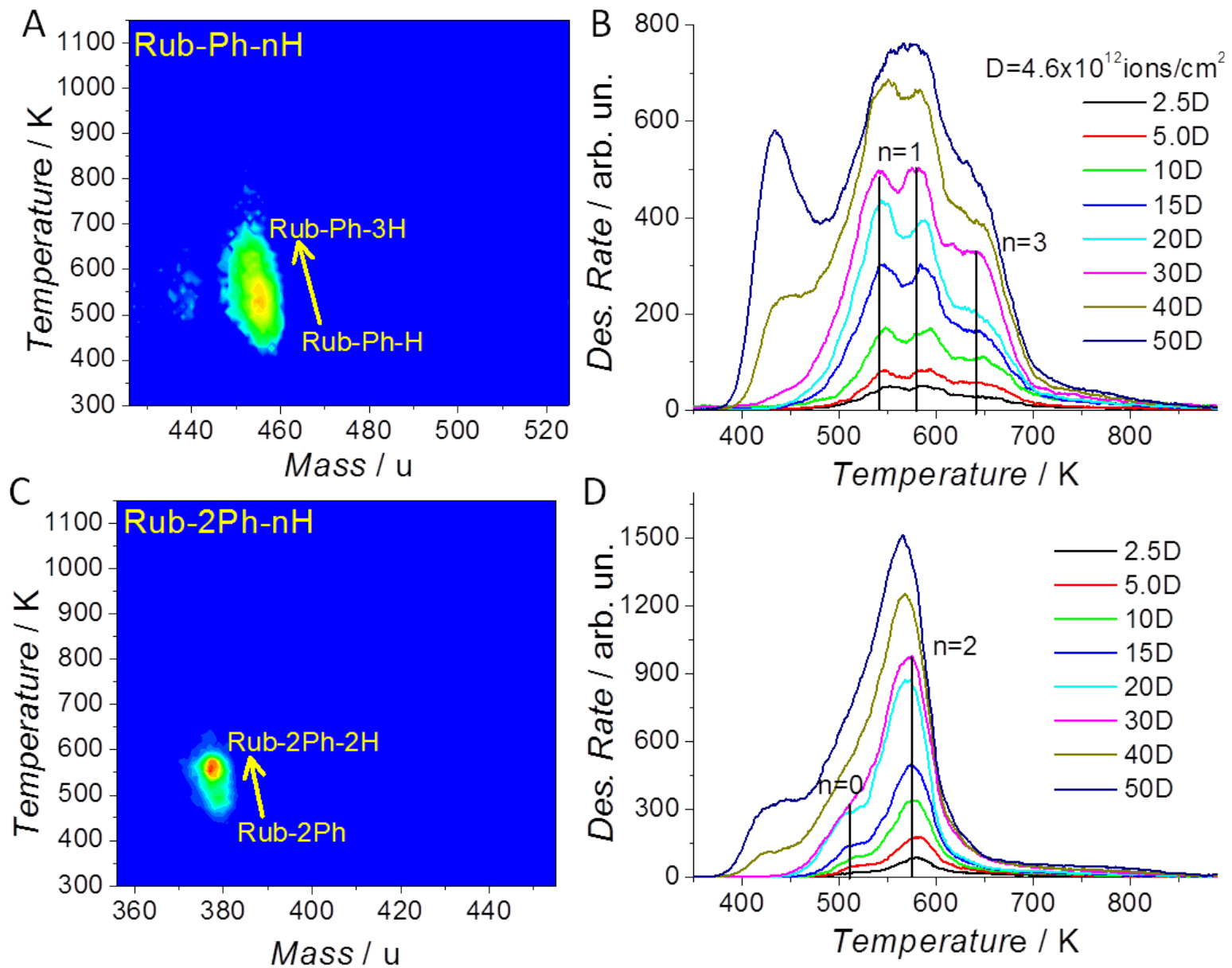

Figure S10: Results of deposition experiments with Phenyl loss fragments of rubrene; A: TRMS map of $1.4 \cdot 10^{13}$ ions $/ \mathrm{cm}^{2}$ Rub-Ph-nH on HOPG, $\beta=4.2 \mathrm{~K} / \mathrm{s}$ with a clear shift from Rub-Ph-H to Rub-Ph- $3 \mathrm{H}$ for higher temperatures; B: TPD coverage variation series and detailed resolution of the components in $\mathrm{A}, \beta=4.3 \mathrm{~K} / \mathrm{s}$ : two Rub-Ph-H and one Rub-Ph-3H components can be resolved; C: TRMS map of $1.4 \cdot 10^{13}$ ions $/ \mathrm{cm}^{2}$ Rub-2Ph-nH on HOPG, $\beta=4.3 \mathrm{~K} / \mathrm{s}$ with a similar shift from Rub-2Ph to Rub-2Ph- $2 \mathrm{H}$ for higher temperature; D: Corresponding TPD coverage variation series, $\beta=4.3 \mathrm{~K} / \mathrm{s}$ : here only one Rub-2Ph and one Rub-2Ph-2H component are observed.

\section{Calculated structures of rubrene Phenyl loss frag- ments}

In figs. $\mathrm{S} 11+\mathrm{S} 12$ all calculated structures for the fragments Rub-Ph-nH and Rub-2Ph-nH are depicted with their relative energies in neutral and cationic charge state. In general, the concerted loss of Phenyl and hydrogen atoms seems to result in the formation of a pentagon.

For Rub-Ph-H both stable isomers are almost equally stable as cations. In the case of Rub-Ph$3 \mathrm{H}$ there is one clearly preferred isomer with two pentagons that still allows a quasiplanar molecular structure.

For Rub-2Ph the most stable structure is the only non-biradical structure we could identify; however, for this isomer a hydrogen transfer reaction in the gas phase is necessary so we are uncertain whether 


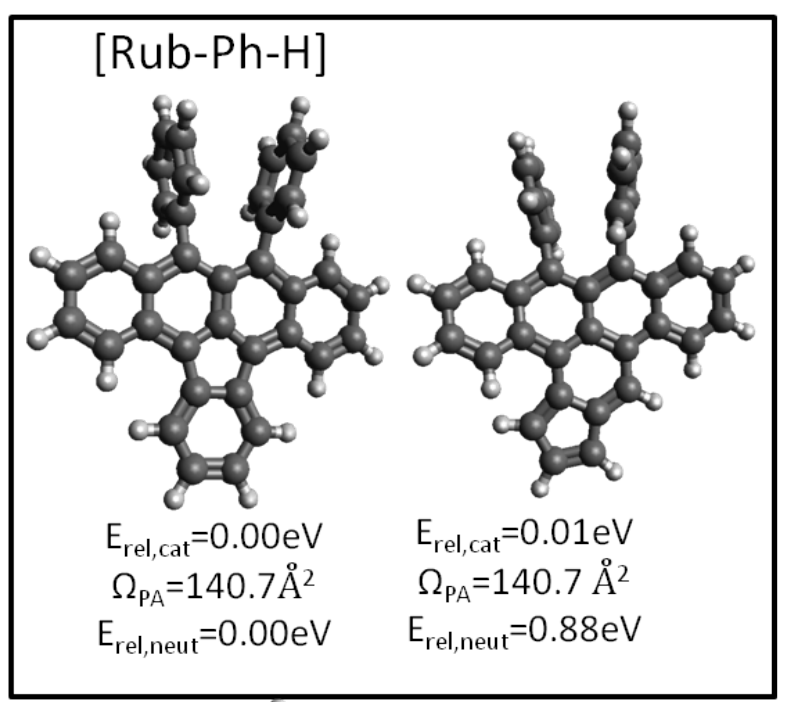

[Rub-Ph-3H]
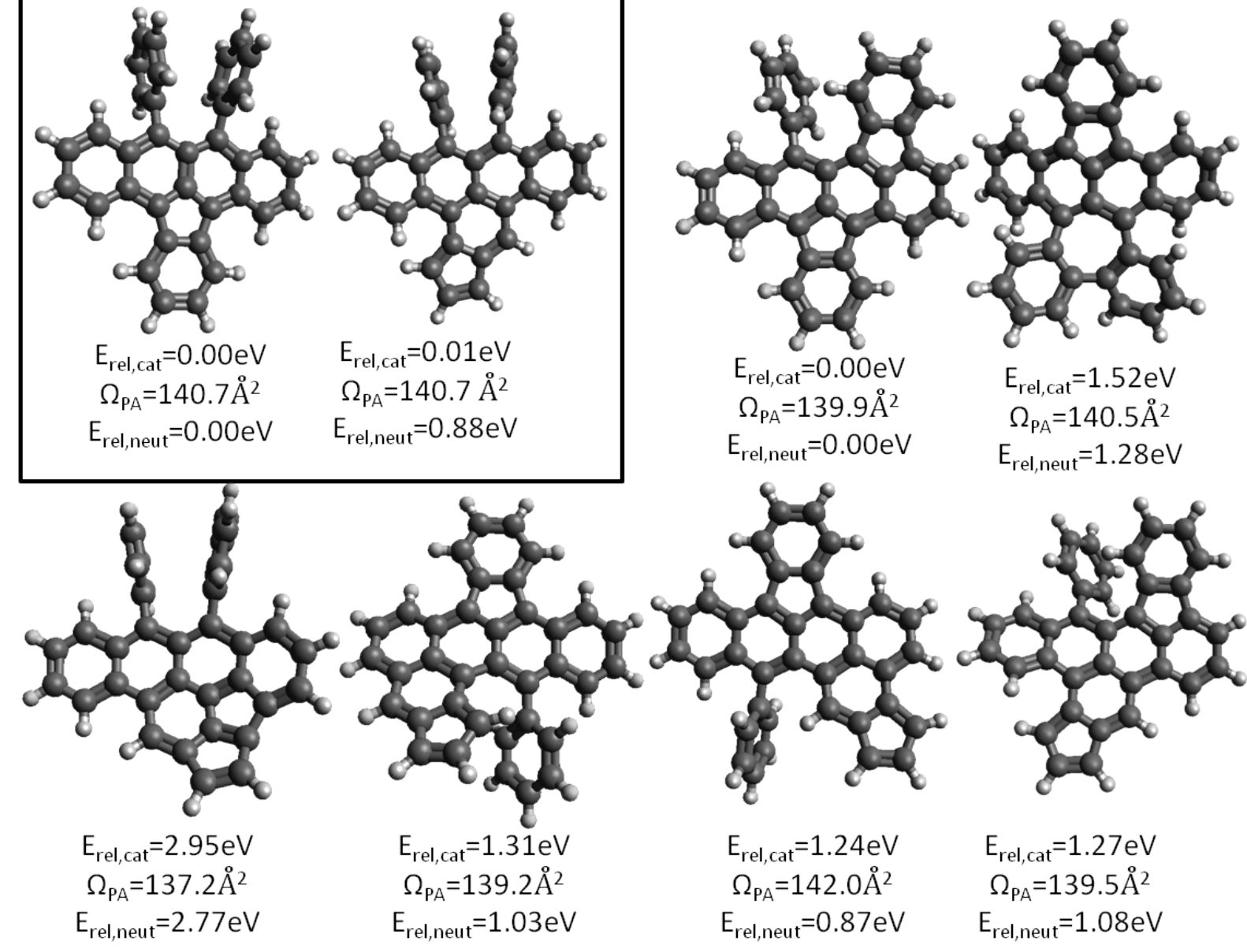

$\mathrm{E}_{\text {rel, cat }}=0.00 \mathrm{eV}$

$\Omega_{\mathrm{PA}}=139.9 \AA^{2}$

$\mathrm{E}_{\mathrm{rel}, \mathrm{cat}}=1.52 \mathrm{eV}$

$E_{\text {rel, neut }}=0.00 \mathrm{eV}$

$\Omega_{\mathrm{PA}}=140.5 \AA^{2}$

$E_{\text {rel,neut }}=1.28 \mathrm{eV}$

Figure S11: Calculated structures of fragments Rub-Ph-nH with their relative stabilities as cations and neutral molecules as well as the cross sections of cations in projection approximation

this species can in fact form. In the case of Rub$2 \mathrm{Ph}-2 \mathrm{H}$ our calculations provided only one stable structure which also looks quite intuitive. 
[Rub-2Ph]

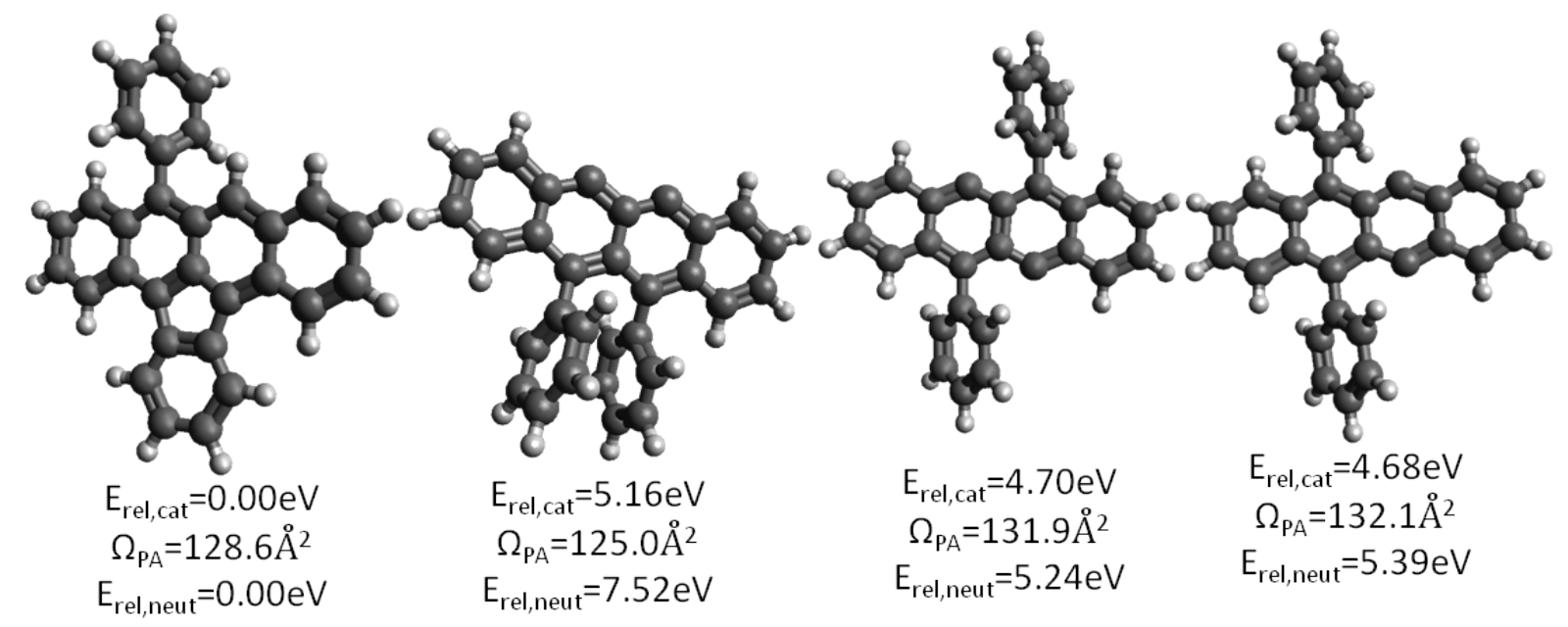

[Rub-2Ph-2H]

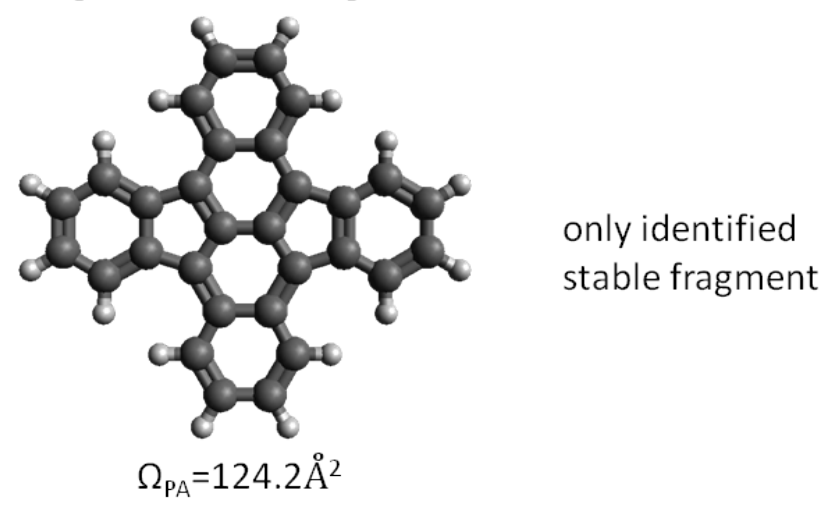

Figure S12: Calculated structures of fragments Rub-2Ph-nH with their relative stabilities as cations and neutral molecules as well as the cross sections of cations in projection approximation 


\section{Mass determination for Pen- $\mathbf{n H}]_{2}$}

Fig. S13 depicts the mass determination of desorbing [Pen-nH]-Dimers from a Pen-nH film as mentioned in section 3.2 of the main paper. The relevant part of the TRMS was extracted and the resulting mass spectrum could be fit with one Gaussian of center mass $548 \mathrm{u}$.

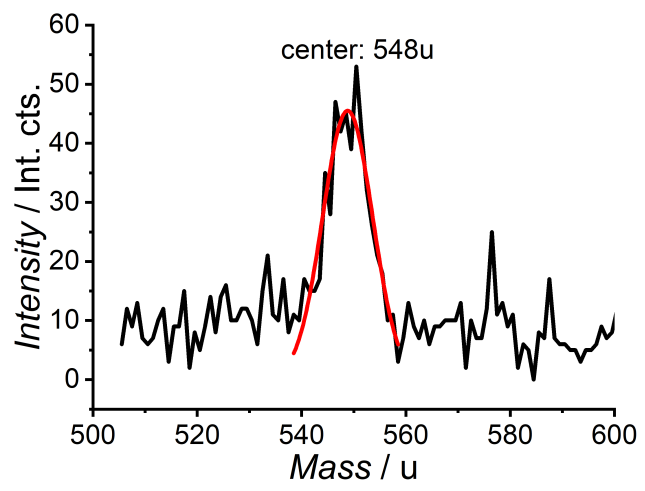

Figure S13: Extracted mass spectrum from a PennH film as mentioned in sec. 3.2 of the main paper. The mass of the desorbing dimer could be determined as $548 \mathrm{u}$.

\section{Merged TRMS map of pen- tacene oligomers}

Complementary to the TRMS map of coronene which is shown in section 3.2 of the main text, a TRMS map of pentacene was also generated and is displayed in fig. S14, clearly showing the desorption of oligomers up to pentamers.

\section{References}

1 H. Horai, M. Arita, S. Kanaya, Y. Nihei, T. Ikeda, K. Suwa, Y. Ojima, K. Tanaka, S. Tanaka, K. Aoshima, Y. Oda, Y. Kakazu, M. Kusano, T. Tohge, F. Matsuda, Y. Sawada, M. Y. Hirai, H. Nakanishi, K. Ikeda, N. Akimoto, T. Maoka, H. Takahashi, T. Ara, N. Sakurai, H. Suzuki, D. Shibata, S. Neumann,
T. Iida, K. Tanaka, K. Funatsu, F. Matsuura, T. Soga, R. Taguchi, K. Saito and T. Nishioka, J. Mass. Spectrom., 2010, 45, 703-714.

2 J. Weippert, J. Hauns, J. Bachmann, A. Böttcher, X. Yao, B. Yang, A. Narita, K. Müllen and M. M. Kappes, J. Chem. Phys., 2018, 149, 194701.

3 J. Weippert, P. Huber, A. Schulz, K. Y. Amsharov, A. Böttcher and M. M. Kappes, submitted to J. Phys. Chem. Lett.

4 A. Becke, Phys. Rev. A, 1988, 38, 3098-3100.

5 S. Vosko, L. Wilk and M. Nusair, Can. J. Phys., 1980, 58, 1200-1211.

6 C. Lee, W. Yang and R. Parr, Phys. Rev. B, 1988, 37, 785-789.

7 A. Becke, J. Chem. Phys., 1993, 98, 5648-5652.

8 F. Weigend, M. Häser, H. Patzelt and R. Ahlrichs, Chem. Phys. Lett., 1998, 294, 143152.

9 K. Eichkorn, F. Weigend, O. Treutler and R. Ahlrichs, Theor. Chem. Acc., 1997, 97, 119124.

10 S. Grimme, J. Antony, S. Ehrlich and H. Krieg, J. Chem. Phys., 2010, 132, 154104.

11 C. Paris, M. Alcamí, F. Martín and S. DíazTendero, J. Chem. Phys., 2014, 140, 204307. 


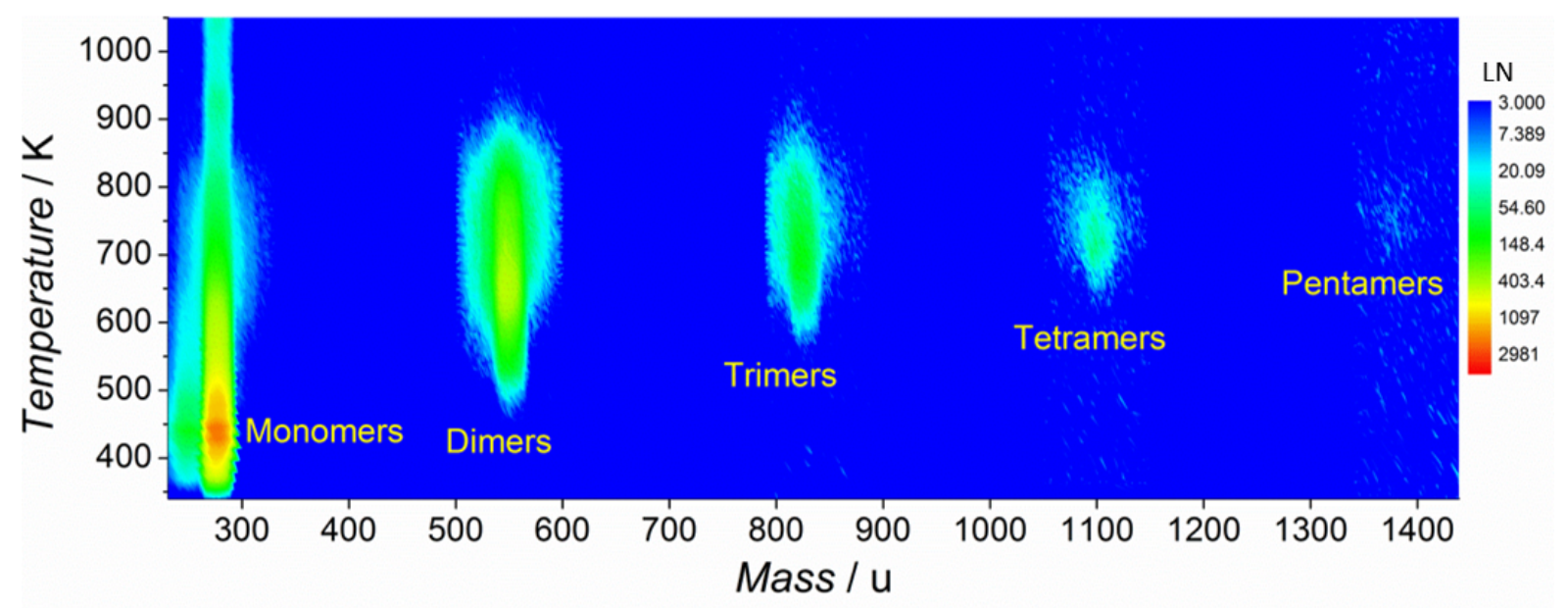

Figure S14: Mass-filter-transmission-corrected, merged TRMS map of $[\mathrm{Pen}-\mathrm{nH}]_{x}(\mathrm{x}=1-5)$ taken from multiple measurements of 20 ML thick mixed films on HOPG 